Supporting Information

\title{
Programmed Multidrug Delivery Based on Bio-Inspired Capsule-Integrated Nanocoatings for Infected Bone Defect Treatment
}

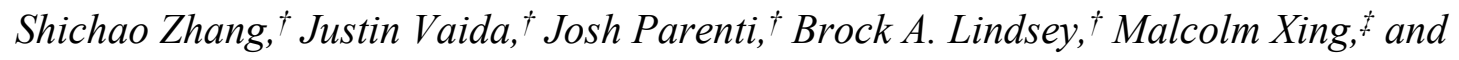

Bingyun $L i *,+$

$\dagger$ Department of Orthopaedics, School of Medicine, West Virginia University, Morgantown, WV, USA

$\$$ Department of Mechanical Engineering, University of Manitoba, and The Children's Hospital Research Institute of Manitoba, Winnipeg, MB, Canada

* Corresponding author. E-mail: bili@hsc.wvu.edu

\section{Supplementary Information contains:}

Supplementary Methods

Supplementary Figures 1-4 


\section{Supplementary Methods}

\section{Drug loading and release measurements.}

The quantitative loading and release of drugs were measured using ELISA kits. In brief, the drug-loaded films were incubated in $2 \mathrm{ml}$ of PBS in a $37{ }^{\circ} \mathrm{C}$ water bath on a rocker. At predetermined time periods, $400 \mu \mathrm{l}$ of medium was taken to measure the release of drugs, and $400 \mu \mathrm{l}$ fresh PBS was added to keep the medium volume constant; the cumulative drug release was then calculated.

\section{Animals and operative procedures.}

In vivo studies were approved by the Institutional Animal Care and Use Committee. SpragueDawley rats were used. The hindlimb to be fractured was shaved first, then a setup was used to produce an open midshaft femur fracture. The thigh was incised through a lateral approach, and the fracture ends were exposed. The rats were infected by injecting $100 \mu \mathrm{l}$ of $10^{2} \mathrm{CFU} / 0.1$ $\mathrm{ml}$ S. aureus directly at the fracture site. Such $S$. aureus concentration led to $90 \%-100 \%$ infection according to our previous studies. The fracture was left open for $1 \mathrm{~h}$ to mimic the "golden hour" of a trauma patient. The fracture was fixed using a Kirschner wire coated with drug-loaded films. The protruding portion of the Kirschner wire was cut off, and the incision closed. After 6 weeks, the rats were euthanized and their tissues were harvested for further studies. The control groups are $\mathrm{L}_{33}$ nanofimls, and the drug-loaded F-I are $\mathrm{L} 5 / \mathrm{C}^{\mathrm{G}} / \mathrm{L} 10 / \mathrm{C}^{\mathrm{L}} / \mathrm{L} 10$

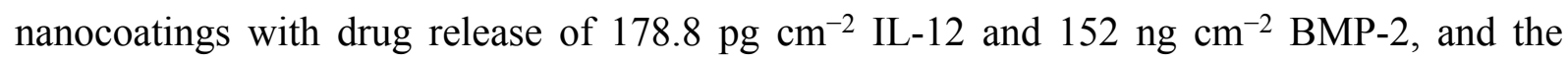
drug-loaded F-II are L5/C $/ \mathrm{L} 5 / \mathrm{C}^{\mathrm{G}} / \mathrm{L} 10 / \mathrm{C}^{\mathrm{L}} / \mathrm{L} 10$ (F-II) nanocoatings with $182 \mathrm{pg} \mathrm{cm}^{-2}$ IL-12 and $286 \mathrm{ng} \mathrm{cm}^{-2}$ BMP-2.

\section{Microbiological evaluations.}

The infection was determined based on $S$. aureus culturing of the surgical tissue samples. For example, the surgical femur was excised postmortem and approximately $500 \mathrm{mg}$ of femur was homogenized in brain heart infusion broth. The ratio of bone to broth was kept at $100 \mathrm{mg}$ to 1 
$\mathrm{ml} ; 0.1 \mathrm{ml}$ of the homogenized bone broth was plated onto blood agar plates and placed in a $37^{\circ} \mathrm{C}$ incubator for $48 \mathrm{~h}$ for further evaluation. Muscle and serum samples were also plated.

\section{Supplementary Figures}

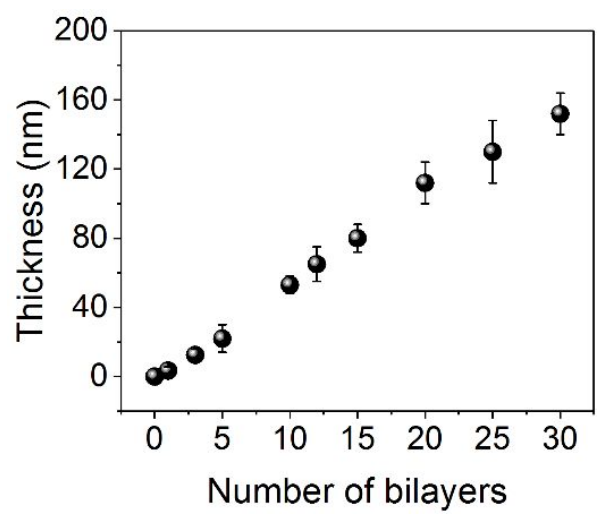

Figure S1. Growth profiles of PL/PG multilayer nanofilms coated on silicon wafers.
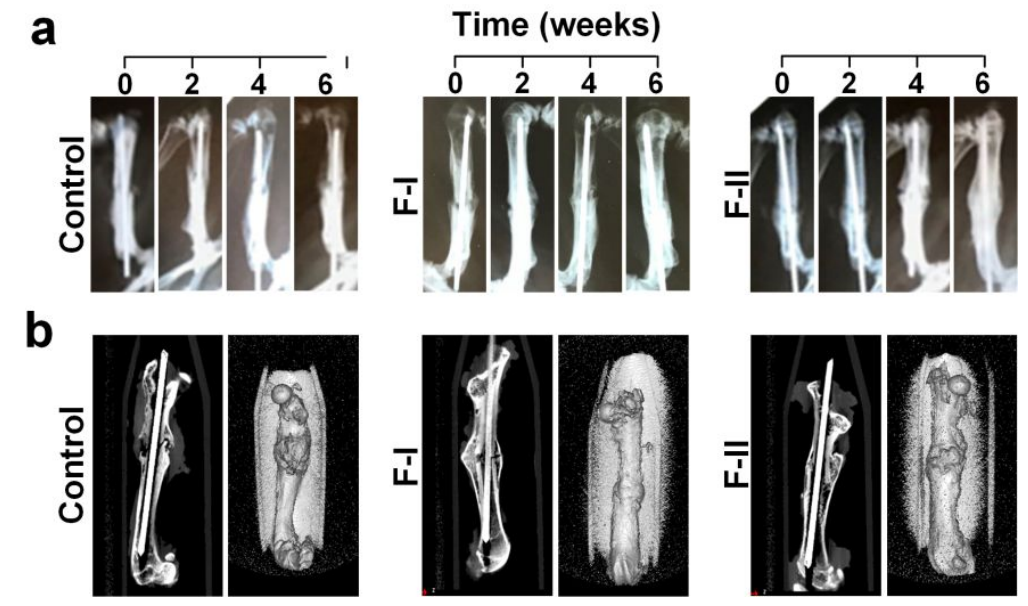

Figure S2. (a) Representative radiographs (0-6 weeks) and (b) 3D micro-CT images (6 weeks) of the fracture callus in rat fracture model after implantation with the control and drug-loaded F-I and F-II nanocoatings. 


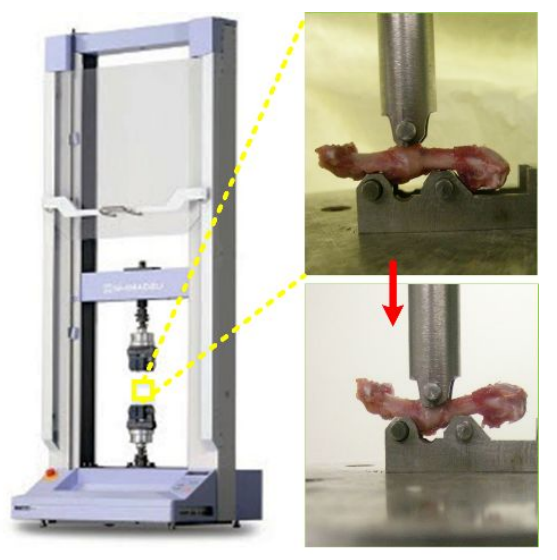

Figure S3. Images of a three-point bending machine and the biomechanical testing of the healing rat femurs.

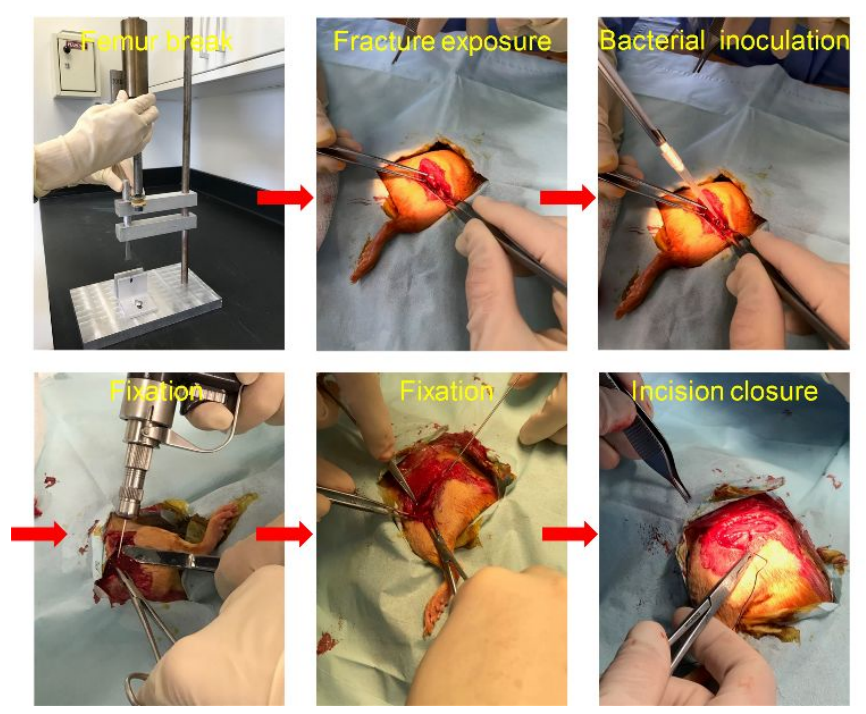

Figure S4. Procedures in creating an open femur fracture and subsequent fixation by nailing intramedullary Kirschner wires coated with nanofilms. 

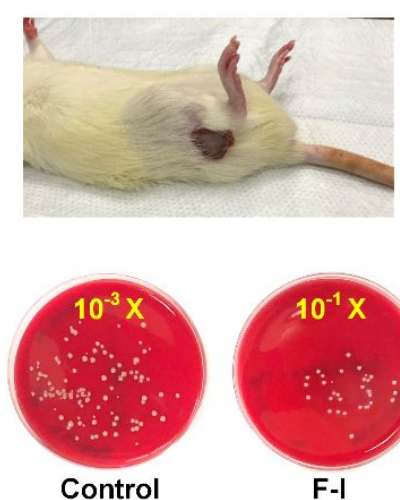

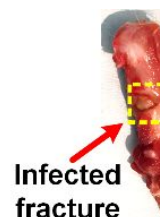

fracture
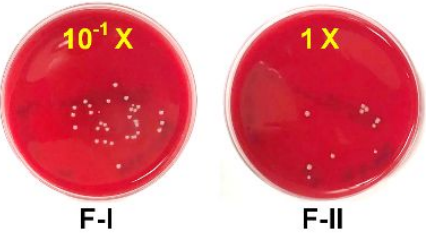

Figure S5. Top, images of infected rat and its bone tissue with infected fracture (top). Bottom, agar plate counting of $S$. aureus for the bone tissue harvested from the control, F-I and F-II groups. 